\title{
Multi-site photometry of the pulsating Herbig Ae star V346 Ori ${ }^{\star}$
}

\author{
S. Bernabei ${ }^{1,2}$, V. Ripepi ${ }^{3}$, A. Ruoppo ${ }^{3}$, M. Marconi ${ }^{3}$, M. J. P. F. G. Monteiro ${ }^{4}$, E. Rodriguez ${ }^{5}$, T. D. Oswalt ${ }^{6}$, \\ S. Leccia ${ }^{3}$, F. Palla ${ }^{7}$, G. Catanzaro ${ }^{8}$, P. J. Amado ${ }^{5}$, M. J. Lopez-Gonzalez ${ }^{5}$, and F. J. Aceituno ${ }^{5}$ \\ 1 INAF - Osservatorio Astronomico di Bologna, via Ranzani 1, 40127 Bologna, Italy \\ e-mail: stefano.bernabei@oabo.inaf.it \\ 2 Departimento de Astrofísica, Universidad de La Laguna, Avda. Astrofisico F. Sánchez sn, 30071 La Laguna, Spain \\ 3 INAF - Osservatorio Astronomico di Capodimonte, via Moiariello 16, 80131 Napoli, Italy \\ e-mail: [ripepi;marconi]@oacn.inaf.it, [ruoppo;leccia]@oacn.inaf.it \\ 4 DMA - Faculdade de Ciências and Centro de Astrofísica da Universidade do Porto, Rua das Estrelas, 4150-762 Porto, Portugal \\ e-mail: Mario.Monteiro@astro.up.pt \\ 5 Instituto de Astrofísica de Andalucía, CSIC, Apdo. 3004, 18080 Granada, Spain \\ e-mail: [eloy;pja;fja;mjlg]@iaa.es \\ ${ }^{6}$ Florida Institute of Technology, Department of Physics and Space Science, 150 W Univ. Blvd., Melbourne, FL 32901-6988, USA \\ e-mail: toswalt@fit.edu \\ 7 INAF - Osservatorio Astrofisico di Arcetri, Largo E. Fermi 5, 50125 Firenze, Italy \\ e-mail: palla@arcetri.astro.it \\ 8 INAF - Osservatorio Astrofisico di Catania, via S. Sofia 78, 95123 Catania, Italy \\ e-mail: gca@oact.inaf.it
}

Received 25 February 2008 / Accepted 21 March 2009

ABSTRACT

\begin{abstract}
Context. The study of pulsation in pre-main-sequence intermediate-mass stars represents an important tool for deriving information about fundamental stellar parameters and internal structure, as well as testing theoretical models. Interest in this class of variable stars has increased significantly during the last decade and about 30 members are presently known in the literature.

Aims. After constructing the frequency spectrum of oscillations in V346 Ori, we apply asteroseismic tools to these data to estimate the intrinsic parameters (mass, luminosity, effective temperature) of V346 Ori and obtain information about its internal structure.

Methods. CCD time-series photometry in the Johnson $V$ filter was obtained for a total of $145.7 \mathrm{~h}$ of observations distributed over 36 nights. The resulting light curves were subjected to a detailed frequency analysis using updated numerical techniques. Photometric and spectroscopic data were also acquired to determine reliable estimates of the stellar properties.

Results. We identified 13 oscillation frequencies, 6 of which with higher significance. These were compared with the predictions of non-radial adiabatic models. The resulting best-fit model corresponds to a mass of $2.1 \pm 0.2 M_{\odot}, \operatorname{luminosity~} \log L / L_{\odot}=1.37_{-0.13}^{+0.11}$, and effective temperature $7300 \pm 200 \mathrm{~K}$. These values are marginally consistent with the association of V346 Ori to Orion OB1a. Alternatively, V346 Ori could be placed at a slightly larger distance than previously estimated.
\end{abstract}

Key words. stars: pre-main sequence - stars: oscillations - stars: individual V346 Orionis - stars: fundamental parameters

\section{Introduction}

Pre-main-sequence (PMS) stars with masses higher than $\sim 1.5 M_{\odot}$ that exhibit variable emission lines are known as Herbig Ae/Be stars (Herbig 1960). Observationally, they are found within star-forming regions and have both variable emission lines (especially $\mathrm{H} \alpha$ ) and strong infrared excess caused by the presence of circumstellar material. In addition, Herbig Ae/Be stars are characterized by photometric and spectroscopic variability on timescales of minutes to years, mainly due to photospheric activity and interaction with the circumstellar environment (e.g., Gahm et al. 1995; Böhm \& Catala 1995).

Although theoretical works have improved our understanding of PMS evolution (see e.g., Palla \& Stahler 1993; D'Antona \& Mazzitelli 1994; Swenson et al. 1994), there remain differences in the models owing to uncertainty in the treatment of convection, of the input physics, and the zero point of the

* Based on data collected with the REM 0.6-m, Loiano 1.5-m, SARA 0.9-m and SNO 1.5-m telescopes. stellar ages. It is therefore desirable to find independent ways of constraining PMS evolutionary tracks and, in turn, the internal structure of intermediate-mass stars, which are subject to dramatic changes during the short-lived PMS contraction phase.

Asteroseismology of Herbig Ae/Be stars can in principle test PMS models by probing their interiors. It is now well established that these stars cross the pulsation instability strip of more evolved stars during contraction toward the main sequence, comprising a class of variable stars called PMS $\delta$ Scuti stars (Kurtz \& Marang 1995; Marconi \& Palla 1998; Catala 2003; Ripepi et al. 2006a).

The first theoretical investigation of the PMS instability strip based on nonlinear convective hydrodynamical models was carried out by Marconi \& Palla (1998, hereinafter MP98), who calculated its topology for the first three radial modes. The subsequent theoretical work by Suran et al. (2001) made a comparative study of the seismology of a $1.8 M_{\odot}$ PMS and post-MS star. These authors found that the unstable frequency range is roughly the same for PMS and a post-MS stars, but that some non-radial $(g)$ modes are very sensitive to the deep internal 
structure. Grigahcene et al. (2006) produced a theoretical instability strip for PMS stars for the first seven radial modes. Since the work by MP98, many new PMS $\delta$ Scuti candidates have been observed and the current census of known or suspected candidates includes about 30 stars (see e.g., Ripepi et al. 2006a; Zwintz 2008). However, only a few of these have been studied in detail (e.g., Ripepi et al. 2003; Böhm et al. 2004; Ripepi et al. 2006b). Thus, the overall properties of this class of variable stars remain poorly determined.

To perform reliable asteroseismological analysis, it is critical to obtain data more accurate than that available in the literature. In particular, it is necessary to measure as many excited oscillations as possible with high precision in frequency and, ideally, absence of aliases. Prior to space data, which will become progressively available from the MOST, COROT and possibly KEPLER satellites, the only way that we can satisfy these requirements is to carry out multi-site observations on promising asteroseismological targets, i.e., stars that are multi-periodic. Starting in 2003, our group has applied the multi-site technique to two targets, V351 Ori (Ripepi et al. 2003) and IP Per (Ripepi et al. 2006b). The observational results obtained for these stars have been interpreted using non-radial adiabatic pulsation theory (see Ruoppo et al. 2007). As a continuation of our ongoing project, here we concentrate on the Herbig Ae star V346 Ori. This object is classified as a member of the Orion OB1a subassociation (Hernández et al. 2006), or of its subgroup centered on 25 Ori (Briceño et al. 2007). It was originally suspected to be variable on a short timescale by Marconi et al. (2000). Subsequently, Pinheiro et al. (2003), on the basis of only two observing nights, confirmed the $\delta$ Scuti nature of the light variation by measuring two highly significant frequencies of pulsation at $f_{1}=35.2 \pm 1.3 \mathrm{c} / \mathrm{d}$ and $f_{2}=22.6 \pm 1.5 \mathrm{c} / \mathrm{d}$ and two less significant frequencies at $f_{3}=45.7 \pm 1.3 \mathrm{c} / \mathrm{d}$ and $f_{4}=18.7 \pm 1.3 \mathrm{c} / \mathrm{d}$. The measured amplitudes of the oscillations in the $V$ band were $A_{1}=2.9, A_{2}=2.8, A_{3}=1.2$, and $A_{4}=1.4 \mathrm{mmag}$. To improve these initial results, we carried out: 1) an extensive photometric multi-site campaign to enlarge the number of observed mode of pulsation; and 2) a spectroscopic analysis to determine the values of $\log g, T_{\text {eff }}$ and $v \sin i$ of V346 Ori; and 3) a comparison with nonlinear pulsation models to constrain the position of V346 Ori in the HR diagram.

The structure of the paper is as follows: in Sect. 2, we describe the observations and data reduction; in Sect. 3, we deal with the frequency analysis; in Sect. 4, we report the results of the spectroscopic analysis; in Sect. 5, we compare the measured frequencies with model predictions and derive an estimate of the parameters of V346 Ori; and in Sect. 6, we provide some final remarks.

\section{Observations and data reduction}

\subsection{Time-series photometry}

Time-series observations were obtained using four different telescopes equipped with CCD devices during 36 nights between 2005, October and 2006, January. A complete description of the observations is given in Table A.1. As a comparison star, we observed HD 287842 (J2000: $05^{\mathrm{h}} 24^{\mathrm{m}} 24.21^{\mathrm{s}}+01^{\circ} 41^{\prime} 33.3^{\prime \prime}$; $V=10.3$, A7). This star was chosen because it is only $\sim 5^{\prime}$ away from V346 Ori and within the field of view of all the instruments used. Its magnitude and color also do not differ too much from those of V346 Ori. The constancy of HD 287842 was checked by comparison with other (fainter) stars present in the CCD frames and in particular with TYC-101-317-1, a

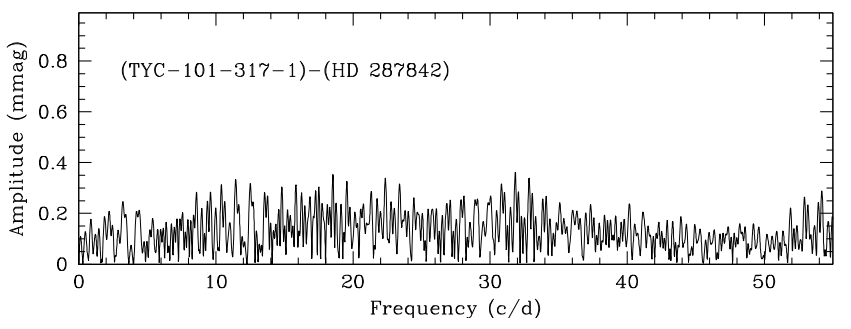

Fig. 1. Fourier analysis of the differential photometry (TYC-101-3171)-(HD 287842). See text.

bright star $(V \sim 11.5 \mathrm{mag})$, which is present in the Sierra Nevada data (five nights, see Table A.1 and below for the data analysis). The Fourier analysis of the differential photometry ${ }^{1}$ (TYC-101-317-1)-(HD 287842) is plotted in Fig. 1. No significant frequency peak is present in the periodogram. The observations were carried out only with the $V$ filter to ensure a time sampling that was sufficiently fast to sample properly the expected rapid pulsations. In the following, we describe the main features of the instrumentation used for this work and provide some details concerning the data reduction and photometric procedures.

- Loiano ${ }^{2}$ : the observations were carried out using the 1.5-m telescope equipped with the BFOSC instrument. The CCD was a EEV $1340 \times 1300$ pixels of individual size $0.58^{\prime \prime}$, and a total field of view of $13^{\prime} \times 13^{\prime}$. The data were reduced following the usual procedures (de-biasing, flat-fielding) and using standard IRAF routines. The aperture photometry was carried out using routines written in the MIDAS environment.

- $\mathbf{R E M}^{3}$ : the data were collected using the 0.60-m automated telescope at La Silla (Chile). Images were obtained with the ROSS spectrograph equipped with a commercial Apogee AP47 camera hosting a Marconi 47-10 1K $\times 1 \mathrm{~K}$ $13 \mu \mathrm{m}$ pitch $\mathrm{CCD}$, which covers $9.54^{\prime} \times 9.54^{\prime}$ with a scale of $0.56^{\prime \prime} /$ pixel. For the data reduction and photometry, we followed the same recipes as for the Loiano observations.

- SARA ${ }^{4}$ (Southeastern Association for Research in Astronomy): we used the 0.9-m automated telescope at Kitt Peak (USA). The images were collected in the $V$ filter using an Apogee AP7p camera with a back-illuminated SITe SIA $502 \mathrm{AB} 512 \times 512$ pixel CCD. The pixels are 24 microns square, corresponding to $0.73^{\prime \prime}$ at the telescope focal plane scale, giving a field of view of $6.2^{\prime} \times 6.2^{\prime}$. Sky flats, dark, and bias exposures were taken every night. The data were calibrated and reduced using standard IRAF routines.

- SNO $^{5}$ (Sierra Nevada Observatory): the data was obtained using the $1.5-\mathrm{m}$ telescope equipped with a $2 \mathrm{k} \times 2 \mathrm{k} \mathrm{CCD}$, with a pixel size of $0.23^{\prime \prime}$ for a total field of view of $7.92^{\prime} \times$ $7.92^{\prime}$. Observations were carried out in the $V$ filter with exposures of about $7 \mathrm{~s}$ (depending on the quality of the night), using a $2 \times 2$ binning. The data were reduced in a standard way by correcting the frames for bias and flat-fielding. Four different apertures were tested of radii 4, 8, 12, and 16 pixels. The 12-pixel aperture (corresponding to about $5^{\prime \prime}$ ) was chosen as the optimal for our observations. The sky inner radius was fixed to 23 pixels and the annulus width to 3 pixels.

\footnotetext{
1 Note that small nightly variations were removed by subtracting for each night the average photometry.

2 http://www .bo.astro.it/loiano/index.htm

3 http://www.rem.inaf.it

4 http://saraobservatory.org

5 http://www.osn.iaa.es
} 

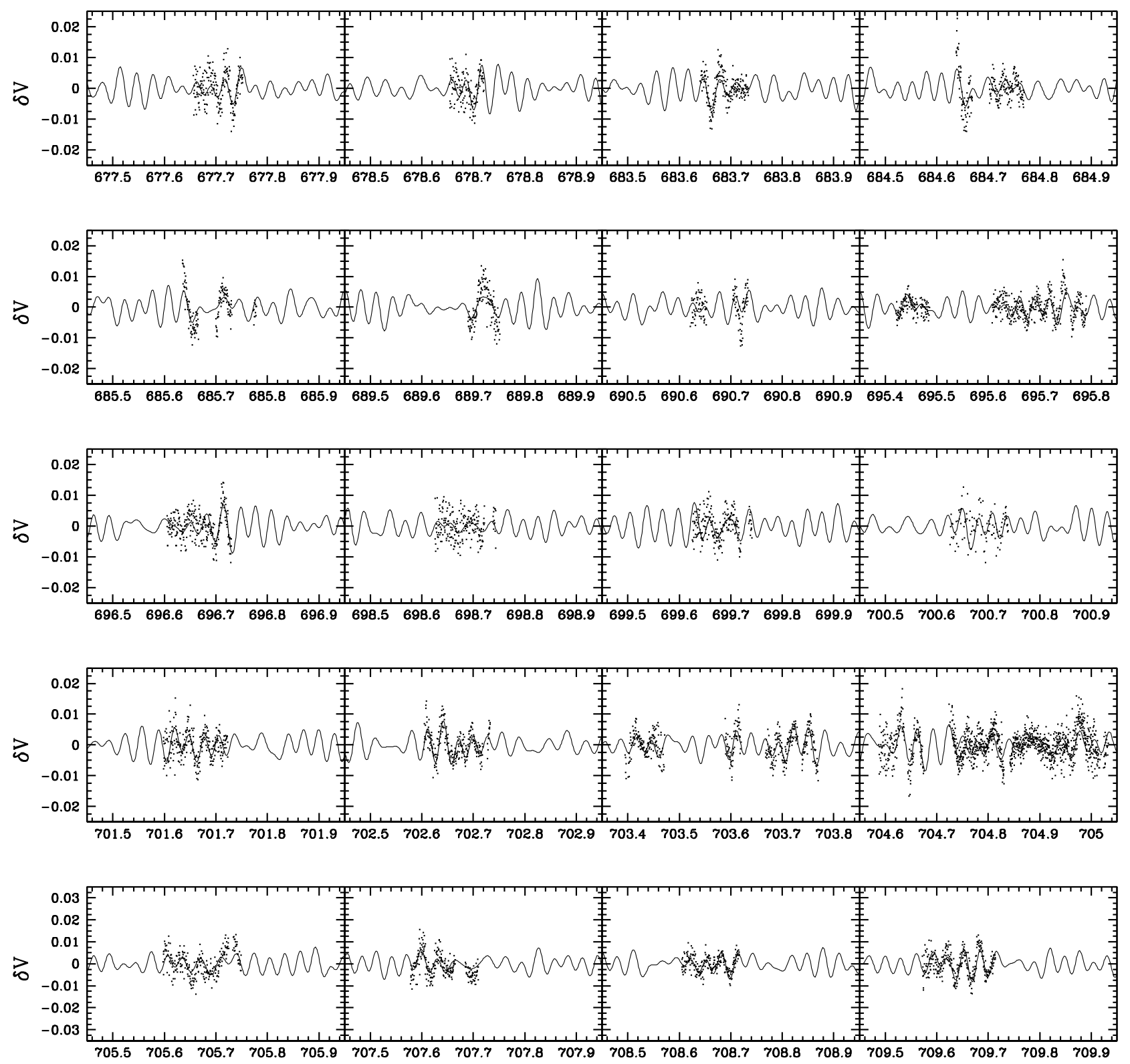

HJD-253000

Fig. 2. Light curve for V346 Ori of the first 20 nights. Note that $\delta V \equiv V_{\mathrm{VAR}}-V_{\mathrm{COMP}}$, where VAR refers to V346 Ori and COMP is the comparison star HD 287842. In all panels, the solid line displays the fit to the data with all the significant frequencies listed in Table A.1 (see text).

In total, we collected $145.7 \mathrm{~h}$ of observations during 36 nights, distributed as follows: $18.7 \mathrm{~h}$ with Loiano; $94.3 \mathrm{~h}$ with REM; 11.2 h with SARA; $21.5 \mathrm{~h}$ with SNO.

To prepare the data for Fourier analysis, we first detrended the data to a common, average zero value. This is needed for the following reasons: 1) to avoid problems caused by differences in the instruments+filters used; 2) to prevent problems with zeropoint differences between different datasets; and 3) to eliminate the long-term photometric variations due to the presence of circumstellar material around the star, a well-known occurrence for Herbig Ae stars such as V346 Ori. The detrending procedure consisted in removing a constant from the photometry for the majority of the nights. In the remaining cases, for nights with a duration of the observations longer than approximately $5 \mathrm{~h}$, we used a linear or quadratic regression to detrend the data. As a consequence of this procedure, we were unable to investigate frequencies lower than about $4.5-5 \mathrm{c} / \mathrm{d}$. The resulting light curve of V346 Ori is shown in Figs. 2 and 3. Here, the solid lines represent the least square fit to the data based on the frequency analysis discussed in Sect. 3.

\subsection{Strömgren-Crawford photometry}

A few $u v b y \beta$ points were also collected for V346 Ori in the Strömgren-Crawford photometric system for calibrating purposes using the six-channel $u v b y \beta$ spectrograph attached to the 0.9-m telescope at Sierra Nevada Observatory. During these measurements, three bright stars C1=HD34888, C2=HD34745, and C3 $=$ HD 36525 were used as check stars, and instrumental magnitude differences were obtained relative to $\mathrm{C} 1$. To transform these instrumental differences into the standard $u v b y \beta$ 

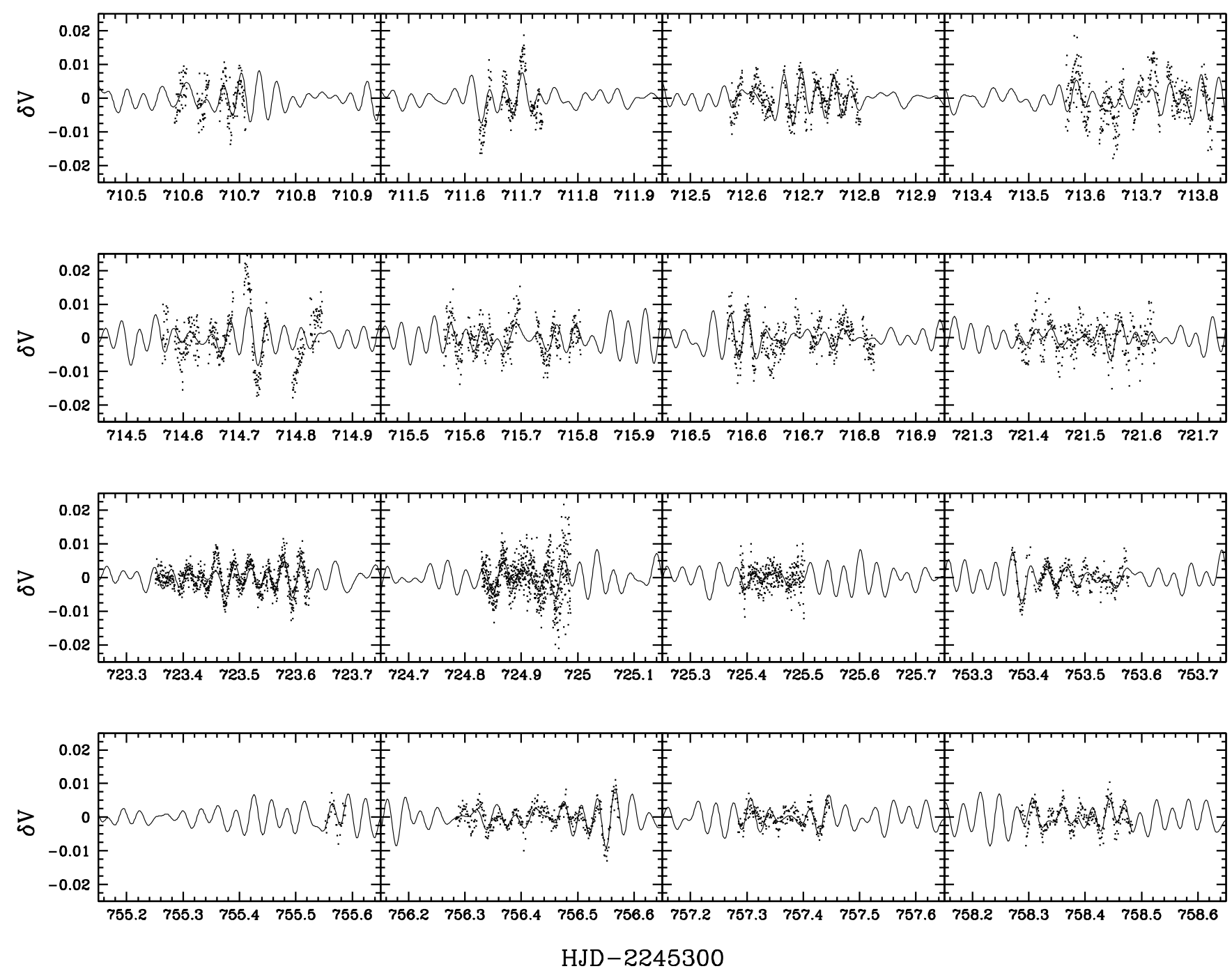

Fig. 3. As in Fig. 2, but for the remaining 16 nights.

system, a set of 19 standards stars were selected from the list of Crawford \& Mander (1966) and Crawford \& Barnes (1970).

Next, the absolute standard $u v b y \beta$ indices for V346 Ori and check stars were obtained following the method described in Rodriguez et al. (2003), using C1 to C3 as zero points. The results are listed in Table 1 together with the values reported in the literature for the check stars. The error bars in this table represent standard deviations in magnitude differences relative to $\mathrm{C} 1$. As seen, our results are in very good agreement with the values found in the homogeneous catalogue of Olsen (1996). Similar results can be found in the lists of Olsen (1983) and Hauck \& Mermilliod (1998).

These indices, together with suitable calibrations available in the literature for $u v b y \beta$, can be used to estimate the physical parameters of V346 Ori using the procedure described in Rodriguez et al. (2001). In particular, a value of $\delta \mathrm{m}_{1}=0.012$ is obtained for the metallicity parameter leading to nearly solar abundances of $[\mathrm{M} / \mathrm{H}]=-0.05( \pm 0.1)$ using the Smalley (1993) relation for metal abundances. Moreover, using the grids of Smalley \& Kupka (1997) for solar abundances, values of $T_{\text {eff }}=$ $7340( \pm 150) \mathrm{K}$ and $\log g=4.0( \pm 0.1)$ are also found, which are in very good agreement with those obtained from spectroscopy and discussed in Sect. 4.

\subsection{Spectroscopic data}

An echelle spectrum for V346 Ori was obtained with the BFOSC instrument at the Loiano Telescope during the night of January 31, 2007. We used the grism \#9 with the cross disperser \#10 to improve the efficiency towards the blue. The resulting spectrum includes 13 orders and covers the wavelength interval from $\sim 3800 \AA$ to $\sim 10000 \AA$. Data reduction was performed using the IRAF package ECHELLE. The resolution of our data was $R \approx 2500$. The spectrum of V346 Ori is shown and discussed in Sect. 4.

\section{Frequency analysis}

The pulsation frequency analysis was carried out with the period04 package (Lenz \& Breger 2005), which adopts both Fourier and least squares algorithms, and permits the simultaneous fitting of multiple sinusoidal variations, and thus does not rely on sequential prewhitening. In addition, period 04 offers the possibility to weight the data during the least squares fitting procedure (see below).

Before presenting the analysis of the time series, we first note that the photometric quality of the data varies among different nights and sometimes during the same night. To account 
Table 1. $u v b y \beta$ indices obtained for V346 Ori and comparison stars.

\begin{tabular}{lrrrrr}
\hline \hline \multicolumn{1}{c}{ Star } & $\begin{array}{c}V \\
(\mathrm{mag})\end{array}$ & $\begin{array}{c}b-y \\
(\mathrm{mag})\end{array}$ & $\begin{array}{c}m_{1} \\
(\mathrm{mag})\end{array}$ & $\begin{array}{c}c_{1} \\
(\mathrm{mag})\end{array}$ & $\begin{array}{c}\beta \\
(\mathrm{mag})\end{array}$ \\
\hline V346 Ori & 10.173 & 0.169 & 0.180 & 0.811 & 2.773 \\
$(5,5)$ & 6 & 6 & 5 & 20 & 10 \\
C1=HD34888 & 6.773 & 0.165 & 0.167 & 0.923 & 2.758 \\
$(6,6)$ & - & - & - & - & - \\
C2=HD34745 & 7.001 & 0.346 & 0.171 & 0.358 & 2.616 \\
(5,5) & 5 & 3 & 4 & 12 & 4 \\
C3=HD36525 & 7.493 & 0.292 & 0.170 & 0.466 & 2.665 \\
(5,5) & 7 & 8 & 4 & 12 & 4 \\
\hline C1=HD34888 & 6.780 & 0.157 & 0.175 & 0.925 & - \\
C2=HD34745 & 7.001 & 0.351 & 0.164 & 0.362 & 2.614 \\
C3=HD36525 & 7.486 & 0.296 & 0.169 & 0.459 & 2.667 \\
\hline
\end{tabular}

The pairs below the star names are the number of points collected for each object in $u v b y$ and $\beta$, respectively. The values given by Olsen (1996) are listed in the bottom part.

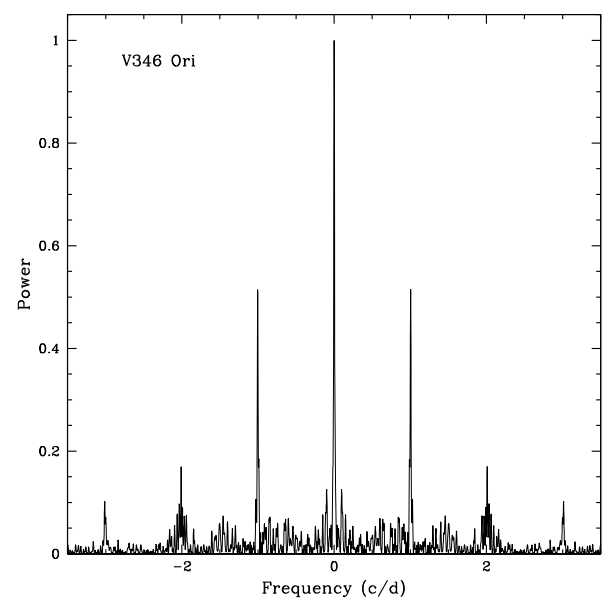

Fig. 4. Spectral window (in power).

for this and include the measured photometric errors based on Poisson statistics, which are often underestimated with respect to the true scatter of the data, we used point-to-point weights in the frequency analysis. Thus, we adopted the weighting scheme in period04 called "deviation weight". Defining residuals as the absolute value of the difference between observed and calculated phase points, this procedure assigns the weights as follows: weight $=1$ for residual $<$ cutoff, and weight $=\left[(\text { cutoff } / \text { residual })^{2}\right]$ for residual $>$ cutoff. The choice of the cutoff value is critical to obtaining reliable results. A general rule is to avoid using a cutoff too small compared to the amplitude of the oscillations (e.g., Handler 2003). We found that a cutoff of 7.5 mmag complies with these requirements and verified that lower values provide unrealistic results, whereas higher values (e.g. cutoff $=1 \mathrm{mmag}$ ) do not produce results that differ significantly from the $7.5 \mathrm{mmag}$ case. As described below, we performed the frequency analysis in two ways: with and without weights.

The frequency analysis requires first the discussion of the Spectral Window (SW), which is shown in Fig. 4. Unfortunately, due to the lack of an observing site in Asia, we were unable to completely remove the $1 \mathrm{c} / \mathrm{d}$ alias. Nevertheless, the $\mathrm{SW}$ of our data appears of good quality in both the weight and no-weight case, since the $1 \mathrm{c} / \mathrm{d}$ is reduced to about $50 \%$ (in power), and the $2 \mathrm{c} / \mathrm{d}$ alias is almost negligible. After this, the procedure consists of the following steps: i) calculation of Fourier transform and identification of the highest amplitude peak; ii) least square fitting to the data with this frequency, improving simultaneously frequency, amplitude, and phase; iii) check of the significance of the extracted frequency; iv) calculation of Fourier transform of the residuals and identification of the new highest amplitude peak; v) least square fitting to the data using simultaneously the derived frequencies; and vi) iteration of the previous steps for the identification of all significant frequencies. To determine whether to accept or reject a frequency, we adopted the widely used requirement of a signal-to-noise $(S / N)$ amplitude equal to 4 (Breger et al. 1993), corresponding to a value of about 12.6 in power (Breger et al. 2005). To calculate the noise level, we again used period 04 to obtain an average value for the noise itself in bins of $10 \mathrm{c} / \mathrm{d}$ width, moving along the spectrum. We verified that changing the size of the bins by up 50\% does not alter the $S / N$ level significantly, especially for the low-amplitude frequencies close to the significance limit.

The result of the frequency analysis is shown in Fig. 5, while the derived frequencies are listed in Table 2 for both the weight and no-weight cases. The differences between the two cases is significant, since the number of derived frequencies varies from 13 in the weighted case to only 6 in the non-weighted case. However, we note that five of the six derived frequencies coincide in both cases, while $f_{6}$ in the non-weighted model is clearly the $-1 \mathrm{c} / \mathrm{d}$ alias of $f_{6}$ in the weighted mode. As seen in Table 2, the use of weights has the effect of reducing the amplitude peaks. More significantly, the noise is also reduced, allowing more frequencies to exceed the $4 \sigma$ threshold albeit only marginally (see $f_{7}$ to $f_{13}$ in Table 2). Given the poor significance of the frequencies derived with our weighting scheme, in the comparison with the models we shall use only the first six frequencies in Table 3. The remaining ones are in any case useful for verifying whether the best-fit model is also able to reproduce them. Finally, we checked if some frequencies could simply be a linear combination of others and verified that all the detected periods are independent of each other.

To determine the errors in the extracted frequencies, we adopted the classical method of calculating the FWHM of the main lobe in the SW, obtaining $\sigma f \sim 0.02 \mathrm{c} / \mathrm{d}$ (see Alvarez et al. 1998, for a discussion of this subject).

We can now compare the present results with those obtained by Pinheiro et al. (2003). As a result, the two significant frequencies seen by Pinheiro et al. (2003) are not in disagreement with our results.

\section{The spectrum of V346 Ori}

\subsection{Empirical determination of atmospheric parameters}

From literature data, we know that V346 Ori is classified as a spectral type A5 or later and has a luminosity class III-V. For our classification, we followed the recipes described by Gray \& Garrison (1989a,b) for early F-type and late A-type stars, complemented with additional criteria by Jaschek \& Jaschek (1987).

From inspection of the spectral region typically used for classification (e.g., Fe I at $\lambda 4046 \AA$; Sr II at $\lambda 4077 \AA$; Fe IITi II at $\lambda \lambda 4172-4179 \AA$; Fe I at $\lambda 4187$ and $4203 \AA$; Fe II at $\lambda 4415 \AA / \lambda 4481 \AA$ (Mg II), we conclude that V346 Ori is of spectral type more similar to A9 than A7. Similarly, we tried to estimate the luminosity class of V346 Ori by analysing the morphology of the blends at $\lambda \lambda 4172-4179 \AA$ and at $\lambda \lambda 4395-$ $4400 \AA$ (Fe II-Ti II-Sc II) with respect to $\lambda 4203 \AA$ and $\lambda 4271 \AA$ 

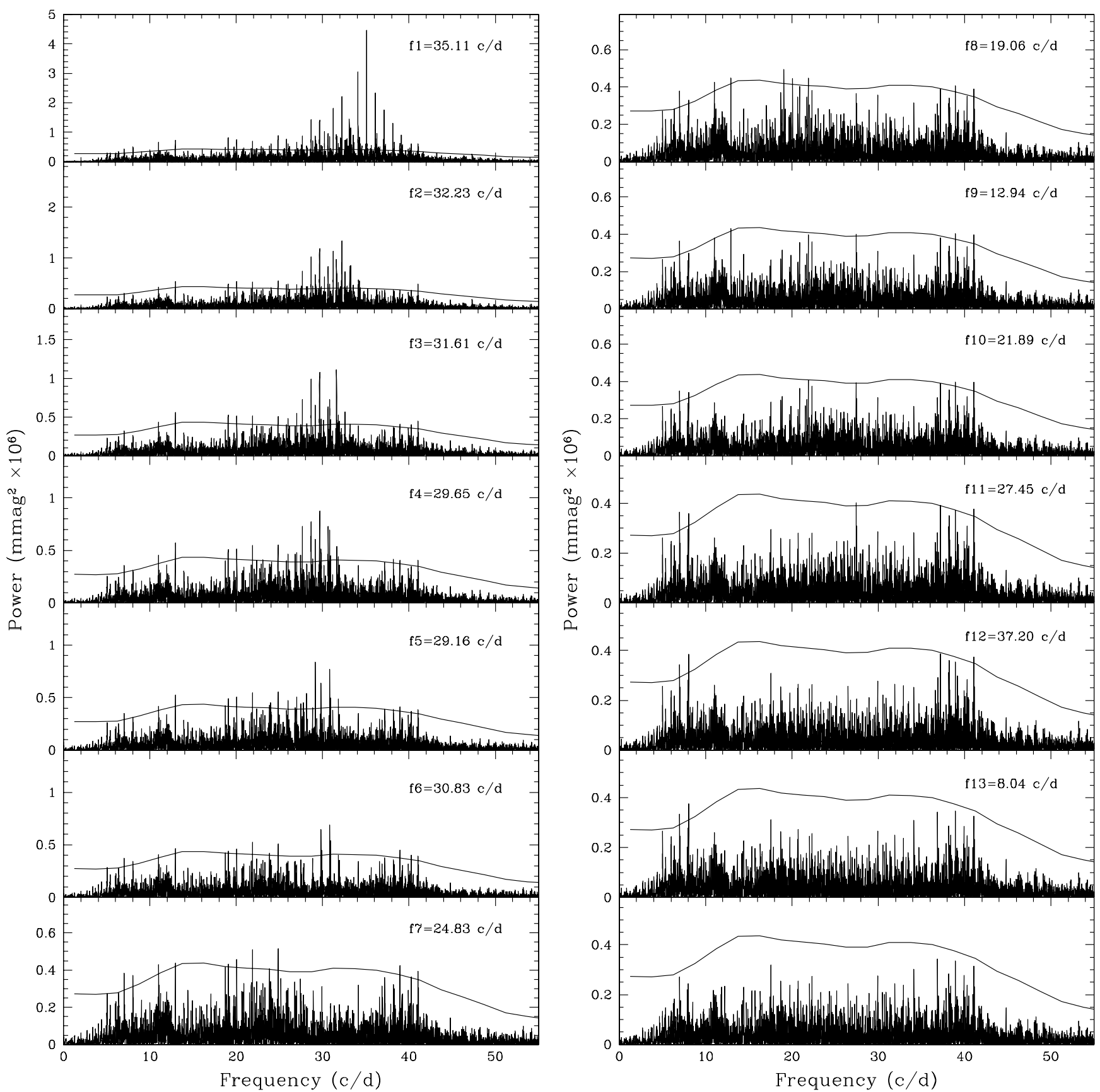

Fig. 5. Frequency analysis for V346 Ori calculated with period 04 adopting the weighting scheme of the data described in the text. The solid line shows the $99.9 \%$ significant level, corresponding to 12.6 times the noise level. In each panel, one peak (i.e., the labelled frequency) is selected and removed from the time series and a new spectrum is obtained. Then, all the derived periodicities are fitted simultaneously to refine frequencies, amplitudes, and phases. The analysis of the residuals produces the subsequent periodogram. The bottom figure in the right panel displays the periodogram after prewhitening with all the significant frequencies.

(Fe I). We computed the ratio $\lambda \lambda 4172-4179 / \lambda 4271$ and $\lambda \lambda 4395-$ $4400 / \lambda 4271$, which is about 2 . This means that V346 Ori luminosity class is IV or V (see Gray \& Garrison 1989a).

The following step was to determine more precisely the spectral type and simultaneously to estimate the rotational velocity $(v \sin i)$ of V346 Ori. To this aim, we used the list of Atype stars with reliable and homogeneous $v \sin i$ published by Royer et al. (2002) coupled with high-resolution spectra from the ELODIE archive (Moultaka al. 2004). In particular, we compared (visually) the spectrum of V346 Ori with several standard stars of spectral type A8 andA9 and luminosity class IV or V, as well as a variety of $v \sin i$. The closest agreement was obtained for HD124675, an A8IV star with $v \sin i \sim 130 \mathrm{~km} \mathrm{~s}^{-1}$ and solar metallicity (Fossati et al. 2008; Bush \& Hintz 2008). Figure 6 shows the superposition of V346 Ori and HD124675 spectra in the region used for spectral classification, as well as the difference between the two spectra. Figure 7 shows the region of $\mathrm{H} \alpha$, which appears to be partially filled and to have emission in the core due to the presence of hot gas around the star, a typical feature of Herbig Ae stars.

We estimate that V346 Ori is an $\operatorname{A8IV}( \pm 1$ spectral class $)$ star with $v \sin i \sim 130 \mathrm{~km} \mathrm{~s}^{-1}$ and approximately solar metallicity. 
Table 2. Frequencies and amplitudes for V346 Ori calculated with period 04 for the two cases, with and without weights (see text).

\begin{tabular}{rrrrr|rccc}
\hline \hline \multicolumn{9}{c}{ Weight case } \\
\hline & $\begin{array}{r}\text { Freq. } \\
(\mathrm{c} / \mathrm{d})\end{array}$ & $\begin{array}{c}\text { Freq. } \\
\mu \mathrm{Hz}\end{array}$ & $\begin{array}{c}\text { Ampl. } \\
(\mathrm{mmag})\end{array}$ & $S / N$ & $\begin{array}{c}\text { Freq. } \\
(\mathrm{c} / \mathrm{d})\end{array}$ & $\begin{array}{c}\text { Freq. } \\
\mu \mathrm{Hz}\end{array}$ & $\begin{array}{c}\text { Ampl. } \\
(\mathrm{mmag})\end{array}$ & $S / N$ \\
\hline$f_{1}$ & 35.107 & 406.33 & 2.23 & 12.1 & 35.107 & 406.33 & 2.20 & 8.8 \\
$f_{2}$ & 32.227 & 373.00 & 1.64 & 9.1 & 32.227 & 373.00 & 1.46 & 5.9 \\
$f_{3}$ & 31.611 & 365.86 & 1.18 & 6.6 & 31.611 & 365.86 & 1.11 & 4.6 \\
$f_{4}$ & 29.652 & 343.19 & 1.22 & 6.9 & 29.651 & 343.19 & 1.33 & 5.5 \\
$f_{5}$ & 29.159 & 337.49 & 0.86 & 4.9 & 29.159 & 337.49 & 1.08 & 4.5 \\
$f_{6}$ & 30.828 & 356.81 & 1.01 & 5.7 & 29.823 & 356.81 & 1.01 & 4.2 \\
$f_{7}$ & 24.833 & 287.42 & 0.82 & 4.6 & & & & \\
$f_{8}$ & 19.058 & 220.58 & 0.77 & 4.1 & & & & \\
$f_{9}$ & 12.936 & 149.72 & 0.77 & 4.1 & & & & \\
$f_{10}$ & 21.886 & 253.31 & 0.75 & 4.0 & & & & \\
$f_{11}$ & 27.446 & 317.66 & 0.78 & 4.5 & & & & \\
$f_{12}$ & 37.203 & 430.59 & 0.74 & 4.0 & & & & \\
$f_{13}$ & 8.045 & 93.11 & 0.69 & 4.0 & & & & \\
\hline
\end{tabular}

The estimated error on frequency is $\sim \pm 0.02 \mathrm{c} / \mathrm{d}(0.23 \mu \mathrm{Hz})$. For the no-weight case, the frequencies are listed in the same order of the weighted mode and not by decreasing amplitude. The horizontal line separates the high significant frequencies from the less significant ones.

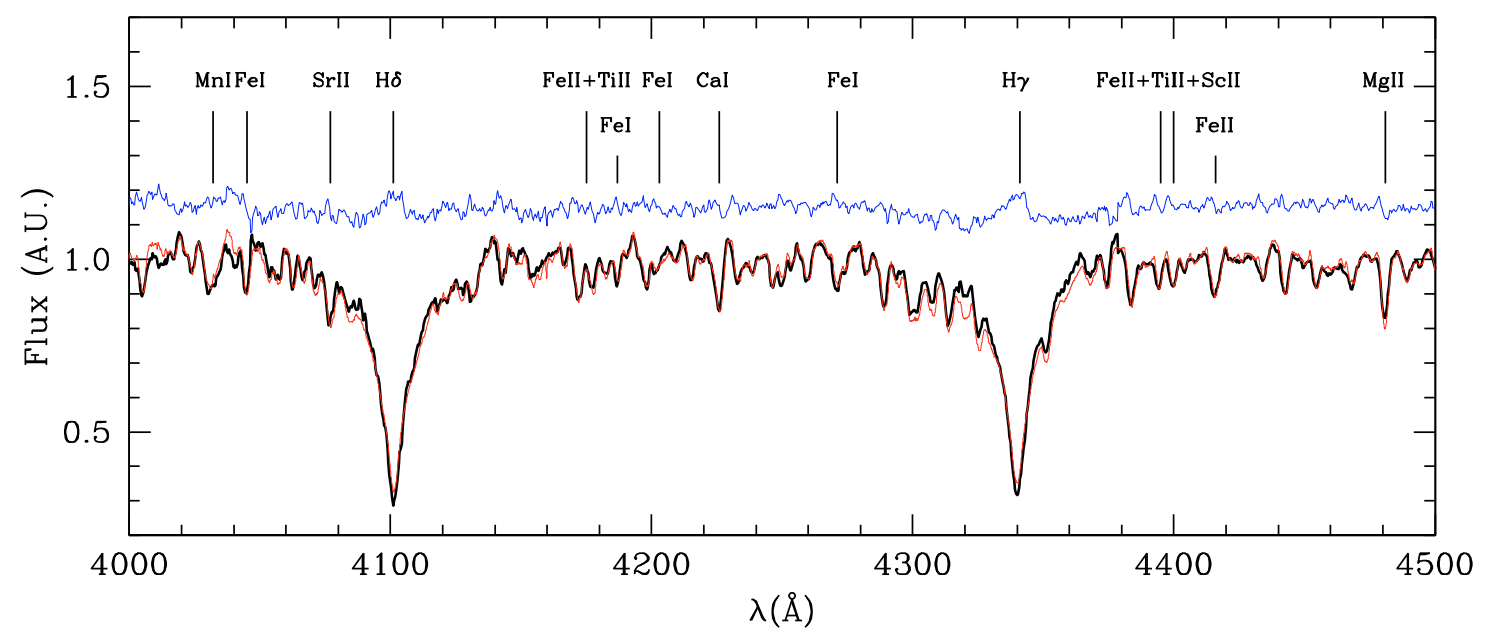

Fig. 6. Selected regions of the normalized V346 Ori spectrum. The black and thin red and blue lines show the data for V346 Ori, for the comparison star HD124675 (A8IV) and the difference between the two spectra, respectively.

This result agrees with some of the previous determinations, but disagrees with others. Considering spectroscopic measurements, V346 Ori has been classified as A2IV (Mora et al. 2001), A5IIIe (Herbig 1960), A7III (Gray \& Corbally 1998), A7III (Blondel et al. 2006), A8 (Valenti et al. 2000), A8V (Vieira et al. 2003), and A9 (Hernández et al. 2006) ${ }^{6}$. Thus, our estimate is consistent with the last three values, and marginally with the A7III evaluation by Gray \& Corbally (1998) and Blondel et al. (2006). It is inconsistent with Herbig (1960) and Mora et al. (2001). The origin of this disagreement in spectral types cannot be easily identified. We suggest two possible causes: 1) the modification of the spectrum by non-photospheric material placed along the line of sight, causing the star to appear earlier in some cases; and 2) the use of different lines/methods for the classification. For example, using $\mathrm{Ca}$ II $\mathrm{K}$ and/or $\mathrm{H}$ lines can often result in earlier spectral-type assignments for this type of star (see Gray \& Garrison 1989a; Gray \& Corbally 1998). According to the

\footnotetext{
${ }^{6}$ Note that some authors do not report the "e" after the spectral classification as is usually done. We verified that this is not due to a lack of detection of emission (in $\mathrm{H} \alpha$ ), but to various circumstances: e.g., the results by Valenti et al. (2000) and Blondel et al. (2006) come from the UV etc.
}

temperature scale of Kenyon \& Hartmann (1995), our estimate for the spectral type of V346 Ori (A8 \pm 1 spectral class) corresponds to $T_{\text {eff }}=7580 \pm 250 \mathrm{~K}$. Similarly, our estimated luminosity class IV-V corresponds approximately to $\log g=3.5-4.3$ (Schmidt-Kaler 1982).

\subsection{Spectral-synthesis determination of atmospheric parameters}

To validate our spectral classification and determine the physical parameters of V346 Ori, we used synthetic spectra.

The approach that we used in this paper was to minimize the difference between the observed and synthetic $\mathrm{H}_{\delta}, \mathrm{H}_{\gamma}$, and $\mathrm{H}_{\beta}$ profiles. As a goodness-of-fit test, we used the parameter:

$\chi^{2}=\frac{1}{N} \sum\left(\frac{I_{\mathrm{obs}}-I_{\mathrm{th}}}{\delta I_{\mathrm{obs}}}\right)^{2}$

where $N$ is the total number of points, $I_{\mathrm{obs}}$ and $I_{\mathrm{th}}$ are the intensities of the observed and computed profiles, respectively, and $\delta I_{\text {obs }}$ is the photon noise. The synthetic spectra were generated in three steps: first, we computed the stellar-atmosphere model by using the ATLAS9 code (Kurucz 1993), then, the stellar 


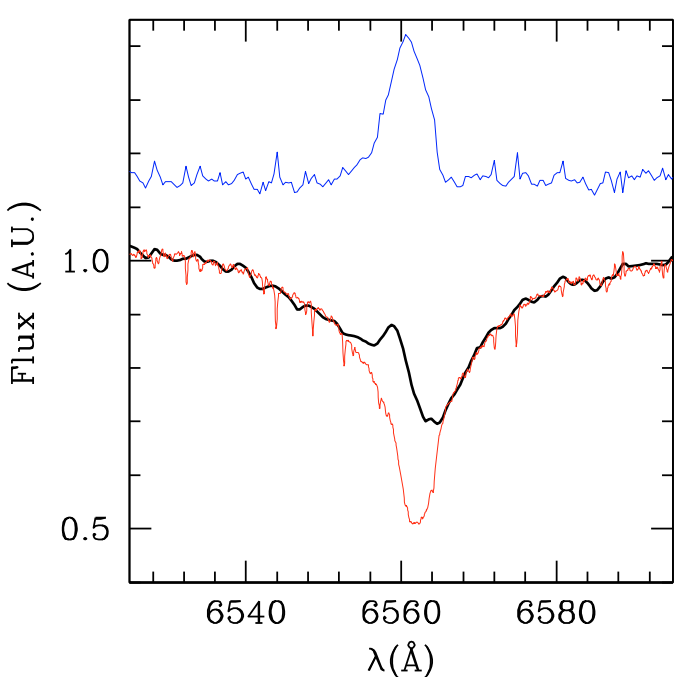

Fig. 7. As in Fig. 6, but in the region of $\mathrm{H}_{\alpha}$. Note the off-center emission present in the difference spectrum.

spectrum was synthesized using SYNTHE (Kurucz \& Avrett 1981), and finally, the instrumental and rotational convolutions were applied. The ATLAS9 code includes the metal opacity by means of distribution functions (ODF), which are tabulated for multiples of the solar metallicity and for various microturbulence velocities.

In this study, we followed the method described in Catanzaro (2008), since the intersection of the three $\chi^{2}$ iso-surfaces is expected to improve the final solution. To decrease the number of parameters, we first computed the $v_{\mathrm{e}} \sin i$ of the star. To derive the rotational velocity, we used SYNTHE, using a guess model computed with the $T_{\text {eff }}$ and $\log g$ estimated in the previous subsection, to reproduce the profile of MgII $\lambda 4481 \AA$ line and obtained a value of $125 \pm 10 \mathrm{~km} \mathrm{~s}^{-1}$, in agreement with our previous estimate of $v_{\mathrm{e}} \sin i \sim 130 \mathrm{~km} \mathrm{~s}^{-1}$. For this star, the best-fit solution was obtained for the model computed with solar ODF for $T_{\text {eff }}=7550 \pm 200 \mathrm{~K}$ and $\log g=3.5 \pm 0.4$.

Although $\mathrm{H}_{\alpha}$ is also present in our spectral range, we did not use this line because of its clearly evident emission at the core. The synthetic line overplotted on the observed one is shown in Fig. 8. It was computed by using the atmospheric parameters derived from the other three Balmer lines. We also shown in the figure for comparison the synthetic spectra with $T_{\text {eff }} \pm \delta T$ and $\log g \pm \delta \log g$.

In conclusion, we have evaluated the atmospheric parameters of V346 Ori by using three different methods: 1) Strömgren-Crawford photometry; 2) empirical determination of spectral and luminosity class; and 3) comparison with synthetic spectra. These approaches provide consistent results within the errors. Given that the comparison with synthetic spectra is the most precise and physically based among the methods we used, in the following we use the atmospheric parameters derived from this approach, but enlarging the error box in $T_{\text {eff }}$ to $\pm 250 \mathrm{~K}$ to take into account the results of methods 1) and 2).

\section{Comparison with models}

After evaluating the physical parameters of V346 Ori, we now proceed to constrain its mass and effective temperature (and, in turn, its luminosity) by comparing the observed frequencies with model predictions. To reduce the number of parameters in the theory versus observations comparison, we decided to

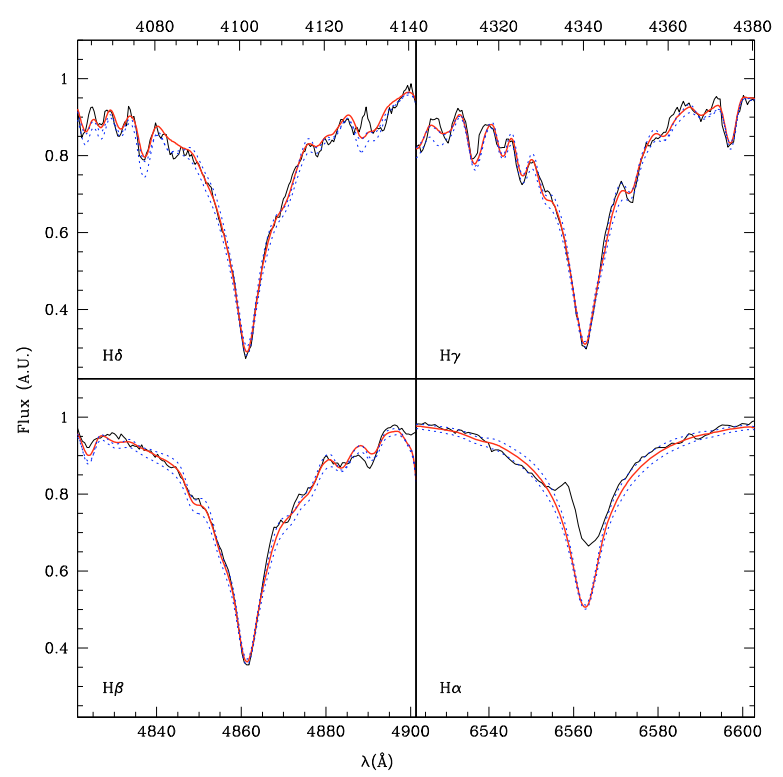

Fig. 8. Zoom of V346 Ori's spectrum in the region of $\mathrm{H}_{\delta}, \mathrm{H}_{\gamma}, \mathrm{H}_{\beta}$ and $\mathrm{H}_{\alpha}$ in comparison with the best-fitting synthetic spectrum $\left(T_{\text {eff }}=7550 \mathrm{~K}\right.$, red line). Also shown in the figure for comparison are the synthetic spectra with $T_{\text {eff }} \pm \delta T$ and $\log g \pm \delta \log g$ (blue dotted lines). Note that only $\mathrm{H}_{\delta}, \mathrm{H}_{\gamma}$ and $\mathrm{H}_{\beta}$ were used for estimating the atmospheric parameter of V346 Ori; $\mathrm{H}_{\alpha}$ is shown for comparison.

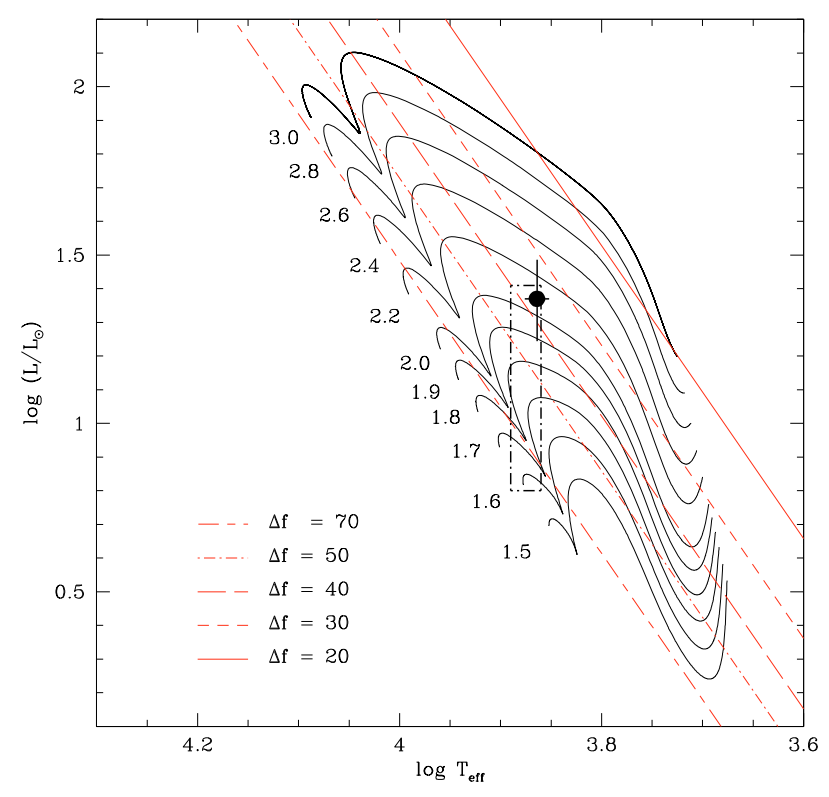

Fig. 9. HR diagram showing PMS evolutionary tracks computed with the CESAM code for the labelled stellar masses (in $M_{\odot}$ ). The dotted lines represent the large separation fit obtained by Ruoppo et al. (2007). The rectangular box delimits the range of luminosity and effective temperature of V346 Ori from the literature. The best fit model is given by the filled dot.

restrict the analysis to the empirically estimated ranges of luminosity $\left(0.8 \lesssim \log L / L_{\odot} \lesssim 1.41\right)$ and effective temperature (7300-7800 K) for V346 Ori. These ranges were derived on the basis of the atmospheric parameters estimated in the previous section and are represented in Fig. 9 by the dot-dashed box, together with the evolutionary tracks computed using the CESAM code (Morel 1997). From the intersection between the box and the evolutionary tracks, we find that the predicted value of the stellar mass lies in the range from $\sim 1.6 M_{\odot}$ to $\sim 2.2 M_{\odot}$. For each 


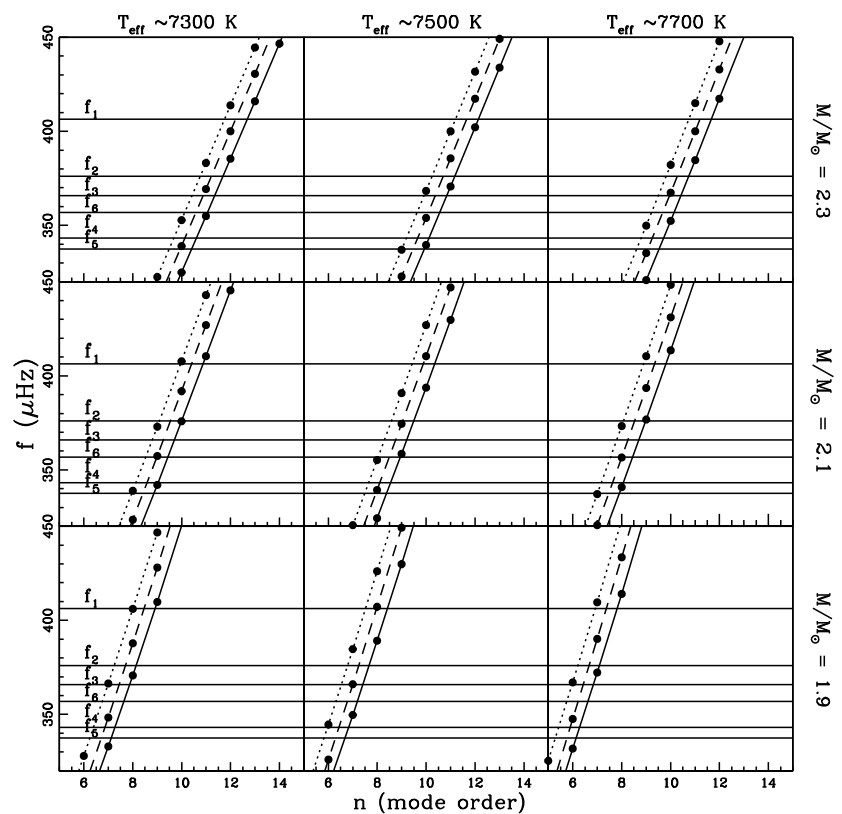

Fig. 10. Predicted frequencies as a function of the radial order $n$. Symbols are connected by a full line in the case of $l=0$, by a dashed line for $l=1$ and by a dotted line for $l=2$. The horizontal lines represent the six most significant frequencies.

stellar mass in this range, we selected three stellar models corresponding to three different effective temperatures, separated by $\sim 200 \mathrm{~K}$, along the corresponding PMS evolutionary track, and performed the pulsation analysis in both radial and non-radial modes $(l=0,1,2)$ using the ADIPLS code ${ }^{7}$. Additional models along the $2.3 M_{\odot}$ were computed to explore the behavior of the predicted frequencies at higher stellar masses. The predicted frequencies, for each selected stellar model, were used to reproduce the six most significant periodicities (from $f_{1}$ to $f_{6}$ ). The comparison between theory and observations is shown in Fig. 10, where the frequencies are plotted as a function of the radial order for three assumptions $l=0,1,2$, by varying the stellar mass and the effective temperature. Inspection of this plot, together with the search for the minimum $\chi^{28}$ suggest that the closest agreement is obtained for $M=2.1 M_{\odot}, \log L / L_{\odot}=1.37$, and $T_{\text {eff }}=7314 \mathrm{~K}$. The position of the best-fit model in the HR diagram is represented by the large dot in Fig. 9. The derived value of the surface gravity of this model is $\log g=3.8$, close to our empirical estimate of $\log g=3.9 \pm 0.4$. The various panels of Fig. 10 show that increasing or decreasing the stellar mass and/or the effective temperatures cause the discrepancies between observed and predicted frequencies to increase. We have also verified that no reasonable match of the observed frequencies is found for stellar masses lower than $M=1.9 M_{\odot}$.

\footnotetext{
7 http://astro.phys.au.dk/ jcd/adipack.n/

8 Given the observed frequencies of V346 Ori, we look for models, into the empirical box of Fig. 9, having $\chi^{2} \leq 1$. As usual, the $\chi^{2}$ is defined as $\chi^{2}=\frac{1}{N} \sum \frac{\left(f_{\mathrm{obs}}-f_{\mathrm{th}}\right)^{2}}{\delta f_{\mathrm{obs}}^{2}+\delta f_{\mathrm{th}}^{2}}$, where $N$ is the total number of observed frequencies, $f_{\mathrm{obs}}$ and $f_{\mathrm{th}}$ are the observed and the theoretical frequencies respectively, and $\delta f_{\text {obs }}, \delta f_{\text {th }}$ are the corresponding errors. If the program does not find any models with $\chi^{2} \leq 1$, that means that not all frequencies are matched within the errors, it discards one-by-one the non-matched frequencies, computes again the $\chi^{2}$ for the remaining frequencies, and searches again for models with $\chi^{2} \leq 1$. The model with the least value of the $\chi^{2}$ is the best-fit model.
}

The comparison between the predicted and observed frequencies in the echelle diagram is presented in Appendix B, confirming that we have found a combination of mass, effective temperature, and luminosity that is able to reproduce in the most accurate way the observed frequencies. We emphasize that this is true only within our explored range of stellar parameters and with the resolution in mass and effective temperature of the current model set.

\section{Final remarks}

We have presented the results of a multi-site campaign on the pulsating Herbig Ae star V346 Ori. The frequency analysis has allowed us to extract six highly significant frequencies on the basis of a no-weight Fourier analysis, and additional seven less significant frequencies by adopting a "deviation weight" scheme. The measured periodicities have been compared with the predictions of non-radial adiabatic models. We found that the resulting best-fit model has a stellar mass of $2.1 \pm 0.2 M_{\odot}$, luminosity $\log L / L_{\odot}=1.37_{-0.13}^{+0.11}, \log g=3.8 \pm 0.2$, and effective temperature of $\sim 7300 \pm 200 \mathrm{~K}$, where the errors are upper limits based on the adopted mass and effective-temperature steps. These estimates agree with the spectroscopic estimates, within the associated uncertainties.

The value of the asteroseismic luminosity is significantly higher than that obtained by other authors. For example, Hernández et al. (2005, H05 hereafter) derived a value of $\log L / L_{\odot}=0.88$ (no errorbar is given) using the average astrometric Hipparcos distance $(\sim 330 \pm 15 \mathrm{pc})$ to the Ori OB1a association and the PMS evolutionary tracks by Palla \& Stahler (1993), assuming $A_{V}=0$ for V346 Ori. However, the uncertainties involved in this calculation are large. Indeed, to derive the luminosity of a star, even knowing the distance, one should know accurately the visual magnitude and the interstellar extinction. This is not straightforward for V346 Ori, which shows a wide variability in the visual band ( $V=9.8-11.8$; Thé et al. 1994), due to the presence of circumstellar material around the star (see e.g., the SED published by Hernández et al. 2006). Similarly the value of $A_{V}$ to be used is not easily achievable: Hernández et al. (2005) used $A_{V}=0$, but the average absorption for the sub-group 25 Ori to which V346 Ori is believed to belong (see Briceño et al. 2007) is $\left\langle A_{V}\right\rangle=0.29$. It is useful to recalculate the $\log L / L_{\odot}$ value for V346 Ori adopting the distance by $\mathrm{H} 05$, but taking into account the value at minimum light for V346 Ori $V=9.8$ (which should be the closest to the photospheric one), and a visual absorption $A_{V}=0.41 \pm 0.1$ as provided by the maps of Schlegel et al. (1998), which are valid in an area of $6.1^{\prime} \times 6.1^{\prime}$ around the star. As a result, allowing for an error of $5 \%$ for the distance and $10 \%$ for both the visual magnitude and absorption, we derive $\log L / L_{\odot}=1.17 \pm 0.11$, which agrees to within $1 \sigma$ with the results of our asteroseismological analysis. Of course, a closer agreement could be obtained by either placing V346 Ori slightly beyond the Orion OB1a association (indeed the distance implied by the present results is $D=400 \pm 50 \mathrm{pc}$, which corresponds to the revised distance of the Orion Nebula Cluster, e.g., Jeffries 2007) or supposing that the extinction due to circumstellar material has been underestimated. In the first case, V346 Ori would not be a member of the Orion OB1a association. To our knowledge, the membership of V346 Ori to OB1a has not been confirmed spectroscopically. To cast light on this complicated scenario, an accurate measurement of the radial velocity of V346 Ori would be highly desirable. 
Acknowledgements. V.R. wishes to thank the personnel of the Loiano Observatory for the competent and kind help during the observations. S.B. acknowledges the REM team for the large amount of observing time allocated for this project and for the professional support during the observations. Partial financial support for this work was provided by PRIN-INAF 2005 under the project "Stellar Clusters as probes of stellar formation and evolution" (P. I. F. Palla). This research has made use of the SIMBAD database, operated at CDS, Strasbourg, France. M.J.P.F.G.M. was supported in part by FCT through project PTDC/CTE-AST/65971/2006 from POCI2010, with funds from the European programme FEDER. T.D.O. was supported by NSF grant AST0206115. P.J.A. acknowledges financial support from a Ramon y Cajal contract of the Spanish Ministry of Education and Science.

\section{Appendix A: Log of the observations}

Table A.1. Log of the observations.

\begin{tabular}{|c|c|c|c|}
\hline $\begin{array}{c}\text { HJD-2450000 } \\
\text { start (days) }\end{array}$ & $\begin{array}{c}\text { HJD-2453000 } \\
\text { end (days) }\end{array}$ & $\begin{array}{l}\text { Duration } \\
\text { (h) }\end{array}$ & Telescope \\
\hline 677.657 & 677.752 & 2.3 & REM \\
\hline 678.654 & 678.721 & 1.6 & REM \\
\hline 683.641 & 683.734 & 2.2 & REM \\
\hline 684.638 & 684.769 & 3.2 & REM \\
\hline 685.635 & 685.779 & 3.4 & REM \\
\hline 689.690 & 689.751 & 1.4 & REM \\
\hline 690.622 & 690.734 & 2.7 & REM \\
\hline 695.421 & 695.486 & 1.6 & Loiano \\
\hline 695.608 & 695.791 & 4.4 & REM \\
\hline 696.605 & 696.730 & 3.0 & REM \\
\hline 698.626 & 698.744 & 2.8 & REM \\
\hline 699.626 & 699.741 & 2.8 & REM \\
\hline 700.626 & 700.738 & 2.7 & REM \\
\hline 701.598 & 701.723 & 3.0 & REM \\
\hline 702.604 & 702.733 & 3.1 & REM \\
\hline 703.395 & 703.472 & 1.8 & Loiano \\
\hline 703.589 & 703.769 & 4.3 & REM \\
\hline 704.587 & 704.675 & 2.1 & REM \\
\hline 704.724 & 705.032 & 7.4 & SARA \\
\hline 705.597 & 705.750 & 3.7 & REM \\
\hline 707.578 & 707.710 & 3.1 & REM \\
\hline 708.605 & 708.717 & 2.7 & REM \\
\hline 709.572 & 709.714 & 3.4 & REM \\
\hline 710.585 & 710.711 & 3.0 & REM \\
\hline 711.625 & 711.738 & 2.7 & REM \\
\hline 712.571 & 712.802 & 5.5 & REM \\
\hline 713.566 & 713.827 & 6.3 & REM \\
\hline 714.563 & 714.847 & 6.8 & REM \\
\hline 715.563 & 715.806 & 5.8 & REM \\
\hline 716.566 & 716.827 & 6.3 & REM \\
\hline 721.376 & 721.624 & 6.0 & Loiano \\
\hline 723.351 & 723.624 & 6.6 & Loiano \\
\hline 725.388 & 725.502 & 2.7 & Loiano \\
\hline 724.829 & 724.987 & 3.8 & SARA \\
\hline 753.369 & 753.577 & 5.0 & OSN \\
\hline 755.552 & 755.588 & 0.9 & OSN \\
\hline 756.284 & 756.573 & 6.9 & OSN \\
\hline 757.284 & 757.446 & 3.9 & OSN \\
\hline 758.284 & 758.483 & 4.8 & OSN \\
\hline
\end{tabular}

\section{Appendix B: Theory versus observations in the echelle diagram}

A powerful tool to investigate the prediction capability of pulsation models is represented by the echelle diagram, where frequencies are reduced to modulo $\Delta f$ by expressing them as $f_{n, l}=f_{0}+\kappa \Delta f+\tilde{f}$ where $\Delta f$ is the large separation, $f_{0}$ is a suitably chosen reference, and $\kappa$ is an integer such that $\tilde{f}$ is between 0 and $\Delta f$. The echelle diagram includes the information about the frequency, large separation, so that the comparison between modeled and observed periodicities in this diagram can allow us to constrain the properties of the frequency spectrum. Hence, for all the models located in the empirical box of Fig. 9, we have used the computed frequencies to build a theoretical echelle diagram and search for one that reproduces most successfully the observed frequencies. As a result, we obtain the best-fit model quoted in Sect. 9. Looking at the behavior of the best-fit model in Fig. 10, we notice that the most significant frequency $f_{1}$ can be reproduced within $\pm 5 \mu \mathrm{Hz}$ either with $l=0$ or with $l=2$. Assuming $l=0$, as also supported by the idea that the largest-amplitude frequency $\left(f_{1}\right)$ is a radial mode, we obtain the echelle diagram shown in the left upper panel of Fig. B.1 for a large frequency separation $\Delta f=35.8 \mu \mathrm{Hz}$. This quantity is evaluated using the analytical relation between the large frequency separation, the stellar luminosity, and the effective temperature derived by Ruoppo et al. (2007). As already found in Fig. 10, the best-fit model is able to reproduce the most significant frequencies to within an uncertainty smaller than $5 \mu \mathrm{Hz}$ apart from $f_{4}$. This frequency is very close to $f_{5}$ and, as discussed by Breger \& Bischof (2002), close frequency pairs should be investigated accurately to establish whether they are real or not.

As already specified, the best-fit pulsation model has been found by requiring it to match only the six most significant frequencies. If the other seven measured frequencies (from $f_{7}$ to $\left.f_{13}\right)$ are added in the echelle diagram, we obtain the plot shown in the left lower panel, where the only deviating frequency, beyond the already noted $f_{4}$, is $f_{12}$.

From our spectroscopic analysis, we have found that V346 Ori has a projected rotational velocity of $v \sin i=125 \pm$ $25 \mathrm{~km} \mathrm{~s}^{-1}$. The effect of rotation on non-radial modes can be roughly estimated using the asymptotic relation for non-radial displacements. This is given by $m f_{\text {rot }}$, where $m$ is the spherical harmonic number and $f_{\text {rot }} \equiv \frac{v \sin i}{2 \pi R \sin i}$ is the rotational frequency. For the inclination angle $i$, we considered the two cases of $i_{1}=\frac{\pi}{4}$ and $i_{2}=\frac{\pi}{2}$, while for the radius we took the value of our best-fit model. Then, with these assumptions, the resulting rotational splitting is $\Delta f_{1} \simeq m 5.3 \mu \mathrm{Hz}$ and $\Delta f_{2} \simeq m 6.2 \mu \mathrm{Hz}$, for $v \sin i=100 \mathrm{~km} \mathrm{~s}^{-1}$ and $\Delta f_{1} \simeq m 7.9 \mu \mathrm{Hz}$ and $\Delta f_{2} \simeq m 9.3 \mu \mathrm{Hz}$ for $v \sin i=150 \mathrm{~km} \mathrm{~s}^{-1}$. It is interesting to note that the displacement of $f_{4}$ and $f_{12}$, from the $l=1$ sequence seems to be consistent with the rotational-splitting estimate for $v \sin i=$ $150 \mathrm{~km} \mathrm{~s}^{-1}$, with these frequencies corresponding to $l=1$, $m= \pm 1$ modes. However, we are aware that this is a very simplified treatment of the rotational splitting phenomenon and that a more accurate analysis by means of asteroseismological models also including rotation is needed to properly test this possibility.

If we discard the assumption that $f_{1}$ corresponds to a radial mode and explore the alternative possibility suggested by the behaviour of the best-fit model in Fig. 10, i.e., that $f_{1}$ is a $l=2$ mode, we obtain the plot shown in the right upper panel of Fig. B.1. In this case, all six frequencies are reproduced to within $\pm 5 \mu \mathrm{Hz}$, apart from $f_{3}$, that deviates a bit more from the $l=2$ sequence. However, if the other seven less significant frequencies are added, we find that theoretical predictions are unable to provide a good match for all the frequencies (see the right lower panel of the same figure). 

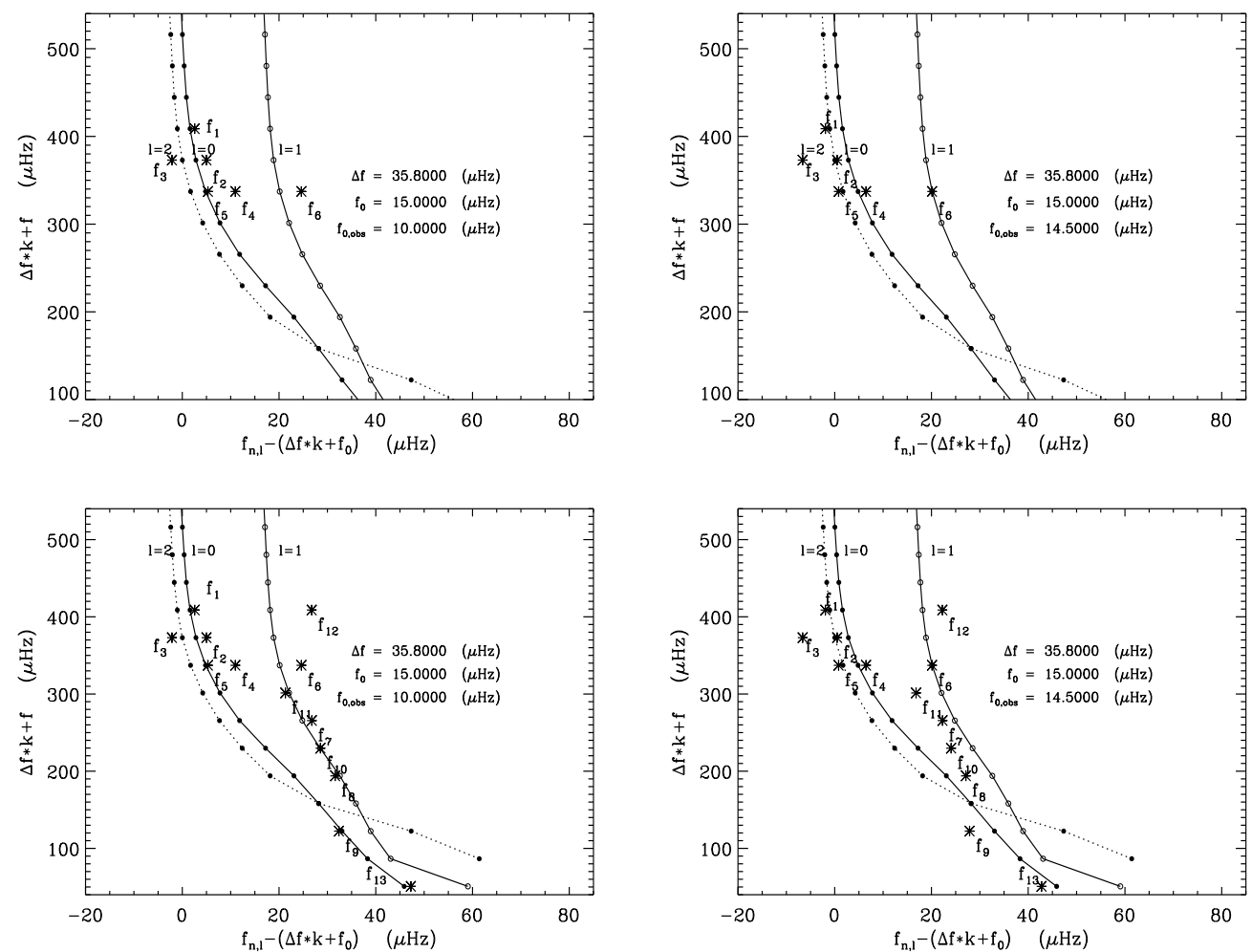

Fig. B.1. Echelle diagram for the model $M=2.1 M_{\odot}, T_{\text {eff }}=7314 \mathrm{~K}$ compared with the frequency data. The parameters adopted to match the data are labelled. Left: with the assumption that the largest amplitude frequency $\left(f_{1}\right)$ is radial $(l=0)$ and by matching only the six most significant frequencies (upper panel) or all the frequencies (bottom panel). Right: with the assumption that the largest amplitude frequency $\left(f_{1}\right)$ is non radial $(l=2)$ and by matching only the six most significant frequencies (upper panel) or all the frequencies (bottom panel).

\section{References}

Alvarez, M., Hernández, M. M., Michel, E., et al. 1998, A\&A, 340, 149 Blondel, P. F. C., Djie, H. R. E., \& Tjin, A. 2006, A\&A, 456, 1045 Böhm, T., \& Catala, C. 1995, A\&A, 301, 155

Böhm, T., Catala, C., Balona, L., \& Carter, B. 2004, A\&A, 427, 907

Breger, M., \& Bischof, K. M. 2002, A\&A, 385, 537

Breger, M., Stich, J., Garrido, R., et al. 1993, A\&A, 271, 482

Breger, M., Lenz, P., Antoci, V., et al. 2005, A\&A, 435, 955

Briceño, C., Hartmann, L., Hernández, J., et al. 2007, AJ, 661, 1119

Bush, T. C., \& Hintz, E. G. 2008, AJ, 1136, 1061

Catala, C. 2003, Ap\&SS, 284, 53

Catanzaro, G. 2008, MNRAS, 385, L33

Crawford, D. L., \& Mander, J. 1966, AJ, 71, 114

Crawford, D. L., \& Barnes, J. V. 1970, AJ, 75, 978

D’Antona, F., \& Mazzitelli, I. 1994, ApJS, 90, 467

Fossati, L., Kolenberg, K., Reegen, P., \& Weiss, W. 2008, A\&A, 485, 257

Gahm, G. F., Loden, K., Gullbring, E., \& Hartstein, D. 1995, A\&A, 301, 89

Gray, R. O., \& Corbally, C. J. 1998, AJ, 116, 2530

Gray, R. O., \& Garrison, R. F. 1989a, ApJ, 69, 301

Gray, R. O., \& Garrison, R. F. 1989b, ApJ, 70, 623

Grigahcene, A., Dupret, M.-A., Garrido, R., Gabriel, M., \& Scuflaire, R. 2006, CoAst, 147, 69

Handler, G. 2003, BaltA, 12, 253

Hauck, B., \& Mermilliod, M. 1998, A\&AS, 129, 431

Herbig, G. H. 1960, ApJS, 4, 337

Hernández, J., Calvet, N., Hartmann, L., \& Briceño, C. 2005, AJ, 129, 856

Hernández, J., Briceño, C., Calvet, N., et al. 2006, AJ, 652, 472

Jaschek, C., \& Jaschek, M. 1987, The classification of stars (Cambridge Univeristy Press)

Jeffries, R. D. 2007, MNRAS, 376, 1109

Kenyon, S. J., \& Hartmann, L. 1995, ApJS, 101, 117

Kurtz, D. W., \& Marang, F. 1995, MNRAS, 276, 191
Kurucz, R. L. 1993, A new opacity-sampling model atmosphere program for arbitrary abundances. In: Peculiar versus normal phenomena in A-type and related stars, ed. M. M. Dworetsky, F. Castelli, \& R. Faraggiana, ASP Conf. Ser. 44, IAU Colloq. 138, 87

Kurucz, R. L., \& Avrett, E. H. 1981, SAO Special Rep. 391

Lenz, P., \& Breger, M. 2005, CoAst, 146, 53

Marconi, M., \& Palla, F. 1998, ApJ, 507, L141

Marconi, M., Ripepi, V., Alcaà, J. M., et al. 2000, A\&A, 355, L35

Mora, A., Merín, B., Solano, E., et al. 2001, A\&A, 378, 116

Morel, P. 1997, A\&A, 124, 597

Moultaka, J., Ilovaisky, S. A., Prugniel, P., \& Soubiran, C. 2004, PASP, 116, 693

Olsen, E. H. 1983, A\&AS, 54, 55

Olsen, E. H. 1996, Priv. Comm.

Palla, F., \& Stahler, S. W. 1993, ApJ, 418, 414

Pinheiro, F. J. G., Folha, D. F. M., Marconi, M., et al. 2003, A\&A, 399, 271

Ripepi, V., Marconi, M., Bernabei, et al. 2003, A\&A, 408, 1047

Ripepi, V., Marconi, M., Palla, F., et al. 2006a, Mem. Soc. Astron. It., 77, 317

Ripepi, V., Bernabei, S., Marconi, M., et al. 2006b, A\&A, 449, 335

Rodríguez, E., López-González, M. J., Rolland, A., Costa, V., \& GonzálezBedolla, S. F. 2001, A\&A, 376, 489

Rodríguez, E., Costa, V., Handler, G., \& García, J. M. 2003, A\&A, 399, 253

Royer, F., Grenier, S., Baylac, M.-O., Gomez, A. E., \& Zorec, J. 2002, A\&A, 393, 897

Ruoppo, V., Marconi, M., Marques, J. P. et al. 2007, A\&A, 466, 261

Schmidt-Kaler, T. 1982, in Landolt-Bornstein, Vol2b Group IV (Springer Verlag) Schlegel, D. J., Finkbeiner, D. P., \& Davis, M., \& Smalley, B. 1993, A\&A, 274, 391

Smalley, B., \& Kupka, F. 1997, A\&A, 328, 349

Suran, M., Goupil, M., Baglin, A., Lebreton, Y., \& Catala, C. 2001, A\&A, 372 , 233

Swenson, F. J., Faulkner, J., Rogers, F. J., \& Iglesias, C. A. 1994, ApJ, 425, 286 Thé, P. S., de Winter, D., \& Pérez, M. R. 1994 A\&A, 104, 315

Valenti, J. A., Johns-Krull, C. M., \& Linsky, J. L. 2000, ApJS, 129, 399

Vieira, S. L. A., Corradi, W. J. B., Alencar, S. H. P. et al. 2003, AJ, 126, 297

Zwintz, K. 2008, ApJ, 673, 1088 